\title{
Muscle spindles of the rat sternomastoid muscle
}

Walter Giuriati (1), Barbara Ravara (1,2), Andrea Porzionato (3), Giovanna Albertin (3), Carla Stecco (3), Veronica Macchi (3), Raffaele De Caro (3), Tiziana Martinello (4), Chiara Gomiero (4), Marco Patruno (4), Dario Coletti (5,6,7), Sandra Zampieri (1,2,8), Alessandra Nori (1)

(1) Department of Biomedical Sciences, Interdepartmental Research Institute of Myology, University of Padova, Padova, Italy; (2) A\&C M-C Foundation for Translational Myology, Padova, Italy; (3) Department of Neurosciences, Section of Human Anatomy, University of Padova, Padova, Italy; (4)Department of Comparative Biomedicine and Food Science, University of Padova, Legnaro, Padova, Italy; (5) Sorbonne Universités, UPMC Univversté Paris 06 (CNRS, UMR 8256, INSERM ERL U1164), Institut Biologie Paris-Seine, Paris, France; (6) Department. of Anatomy, Histology, Forensic Medicine \& Orthopaedics, School of Medicine Sapienza University of Rome, Rome, Italy; (7 )Interuniversity Institute of Myology, 00185 Rome, Italy; (8) Physiko- und Rheumatherapie, St. Poelten, Austria

This article is distributed under the terms of the Creative Commons Attribution Noncommercial License (CC BY-NC 4.0) which permits any noncommercial use, distribution, and reproduction in any medium, provided the original author(s) and source are credited.

\begin{abstract}
The sternomastoid (SM) muscle in rodents presents a peculiar distribution of fiber types with a steep gradient from the ventral, superficial, white portion to the dorsal, deep, red region, where muscle spindles are restricted. Cross section of the medial longitudinal third of the rat SM contains around 10,000 muscle fibers with a mean diameter of 51.28 $\pm 12.62(\mu \mathrm{m}+/-\mathrm{SD})$. Transverse sections stained by Succinate Dehydrogenase (SDH) reaction clearly presents two distinct regions: the dorsal deep red portion encompassing a $40 \%$ cross section area contains a high percentage of packed SDH-positive muscle fibers, and the ventral superficial region which contains mainly SDH-negative muscle fibers. Indeed, the ventral superficial region of the rat SM muscle contains mainly fast $2 \mathrm{~B}$ muscle fibers. These acidic ATPase $\mathrm{pH}$ 4.3-negative and SDHnegative 2B muscle fibers are the largest of the SM muscle, while the acidic ATPase $\mathrm{pH} 4.3$ positive and SDH-positive Type 1 muscle fibers are the smallest. Here we show that in thin transverse cryosections only 2 or 3 muscle spindle are observed in the central part of the dorsal deep red portion of the SM muscle. Azan Mallory stained sections allow at the same time to count the spindles and to evaluate aging fibrosis of the skeletal muscle tissue. Though restricted in the muscle red region, SM spindles are embedded in perimysium, whose changes may influence their reflex activity. Our findings confirm that any comparisons of changes in number and percentage of muscle spindles and muscle fibers of the rat SM muscle will require morphometry of the whole muscle cross-section. Muscle biopsies of SM muscle from large mammals will only provide partial data on the size of the different types of muscle fibers biased by sampling. Nonetheless, histology of muscle tissue continue to provide practical and low-cost quantitative data to follow-up translational studies in rodents and beyond.
\end{abstract}

Key Words: rat, sternomastoid muscle, muscle spindles, fiber types, translational studies

Eur J Transl Myol 28 (4): 376-385, 2018

The sternomastoid (SM) muscle in rodents has a peculiar distribution of muscle fiber types, presenting macroscopically a distinct ventral superficial white portion, dominated by fast-glycolytic muscle fibers (Type 2B), and a dorsal red portion where the fastoxidative-glycolytic muscle fibers (type 2A) are mainly present, together with slow-oxidative muscle fibers (Type 1). ${ }^{1,2}$ This kind of regional distribution of fiber types may occur also in leg muscles (e.g., tibialis anterior and EDL muscles), but not at the extreme extent as detected in the SM muscle. ${ }^{3,4,5}$ This peculiar regional distribution of muscle fiber types suggests a functional specialization of the different portions, despite common terminal cranial tendon and an unique nerve. Given the peculiar nature of this muscle, we further characterize their regional distribution by histological and 
histochemical analyses of transverse sections of the longitudinal medial third portion of the SM muscle. About twenty muscle spindles are homogeneously distributed along the whole muscle mass of the rat $\mathrm{SM},{ }^{1,2}$ and we highlight their position in our transverse sections. Limits to characterization of this muscle in large mammals by muscle biopsy morphometry are discussed. ${ }^{6}$

\section{Materials and Methods}

Animals and muscles.

The SM muscles were harvested from adult female Wistar rats $(300 \mathrm{~g})$. The animals were housed in the vivarium of the Department of Neurosciences (University of Padova) where they were kept in cages within a $24 \pm 2{ }^{\circ} \mathrm{C}$ room on a 12-hour light/dark cycle, and offered food and water ad libitum. All procedures in this study were performed according to the international ethical principles of animal experimentation and approved by the Ethics on Animal Experimentation Committee of the University of Padova. Animals were humanely killed with deep anaesthesia (according to the protocol) and then muscles were excised, weighed, snapfrozen in liquid nitrogen $\left(-196^{\circ} \mathrm{C}\right)$, and stored at $-80^{\circ} \mathrm{C}$ until use. Serial cross sections $(10 \mu \mathrm{m})$ were cut from the medial third of four SM muscles using a cryostat microtome at $-25^{\circ} \mathrm{C}$. Sections were mounted on polylisine-treated glass slides and air dried.

Histological stainings

Muscle cryosections were then stained with either standard Azan-Mallory procedure or by histochemical methods to determine muscle fiber types. Heidenhain's Azan modification of Mallory's triple staining. For highlight the collagen fibers from the connective tissue of the rat SM normal muscle serial sections were incubated $15 \mathrm{~min}$ in 4\% PFA at room temperature, rinsed in distilled water $2 \times 5 \mathrm{~min}$, and stained in azocarmine $\mathrm{G}$ solution, warmed up to about $56^{\circ} \mathrm{C}$ for $60 \mathrm{~min}$. Sections were rinsed in distilled water, $3 \times 1 \mathrm{~min}$ and differentiated in $0,1 \%$ anilin-Et-OH until only nuclei, are distinctly stained thorough rinse in Et-OH-acid acetic to stop differentiation and remove anilin. Sections were mordonted in $5 \%$ phospotungstic acid solution, for 60 min and were brief rinsed in distilled $\mathrm{H} 2 \mathrm{O}$. Sections were stained in anilin blu-orange $\mathrm{G}$ solution for $60 \mathrm{~min}$ and rinsed in distilled $\mathrm{H} 2 \mathrm{O}, 3 \times 1 \mathrm{~min}$ and dehydrated in graded alcohol solutions (ethanol 70, 90, 100\%) cleared in xylene and mounted in permanent medium: Canada balsam. Azocarmine staining solution: dissolve $0,1 \mathrm{~g}$ azocarmine $\mathrm{G}$ in $100 \mathrm{ml}$ boiling distilled H2O. Cool down, add $1 \mathrm{ml}$ glacial acid acetic. Anilin-Et-OH differentiation solution: Et-OH acetic acid rinsing fluid: Et-OH 96\%: $1000 \mathrm{ml}$; Glacial acetic acid: $10 \mathrm{ml}$. Mordating solution: distilled water: $1000 \mathrm{ml}, 5 \%$ phosphotungstic acid. Anilin blu-orange $G$ staining solution: distilled H2O: $1000 \mathrm{ml}$; orange G: $20 \mathrm{~g}$; anilin blu: $5 \mathrm{~g}$; glacial acetic acid: $80 \mathrm{ml}$. Bring to the boil and cool down after dissolution of the dyes and filter.
Succinate Dehydrogenase (SDH) staining.

Succinate Dehydrogenase (SDH) staining was performed to distinguish between oxidative and non-oxidative (or "less" oxidative) muscle fibers. Serial cross-sections from SM muscles were incubated for $60 \mathrm{~min}$ at $37^{\circ} \mathrm{C}$ in SDH incubation solution $(0.1 \%$ nitro blue tetrazolium [NBT] in 0.1M phosphate buffer [pH 7.2-7.6] containing $0.1 \mathrm{M}$ sodium succinate) and then rinsed in distilled $\mathrm{H} 2 \mathrm{O}$ ( 3 changes $x 1 \mathrm{~min}$ ). To remove unbound NBT, the sections were incubated in 3 changes ( 1 min per change) of acetone in water solutions $(30,60$, and $90 \%$ acetone) in first increasing and then decreasing order of acetone concentration. Sections were rinsed in distilled $\mathrm{H} 2 \mathrm{O}$ for 3 changes ( 1 min each), dehydrated in graded ethanol solutions (i.e., 70, 90, and 100\%), cleared in xylene, and finally mounted in permanent medium (Canada Balsam). staining myofibrillar actomyosin ATPase

Myofibrillar actomyosin ATPase staining. Two different procedures were used for staining myofibrillar actomyosin ATPase following the methods described by Brooke and Kaiser ${ }^{7,8}$ and by Guth and Samaha., ${ }^{9,10}$

Histological Morphometry. Muscle fiber size and the absolute number or percent composition of different muscle fiber types were determined in transverse cryosections of the SM muscle. Quantitative evaluations were performed using images of stained cryosections with Scion Image software for Windows version Beta 4.0.2 (2000 Scion Corporation). Tissue type distribution was determined using Adobe Photoshop software (Adobe Systems Incorporated, San Jose, CA).

\section{Results and Discussion}

Transverse sections of rat SM stained by SDH reaction (Fig.1A) clearly shows two distinct regions: 1) a part of the section (around $40 \%$ of the area) that contains high abundance of SDH positive myofibers (right part of Fig. 1A); and 2) a part of the section (around 60\% of the area) that reveals a more checker-board appearance. In the latter the number of SDH positive muscle fibers per area unit decreases dramatically. The right side of the section, that is the one peculiarly rich in SDH positive muscle fibers, corresponds to the dorsal deep red part of the SM muscle, while the left part of the figure to the ventral superficial white region of the rat SM muscle, revealing a dorsal (rihgt) to ventral (left) gradient of muscle fiber types. . In the sections harvested from the medial third of the rat SM muscle (Figure 1), we counted around 10,000 muscle fibers (Table 1). Tables 1 and 2 show that SDHpositive muscle fibers are $73 \%$ of the total in the SM deep region. In the superficial region (left part of the cryosection in Fig. 1A), the SDH staining pattern indicates that only $31.1 \%$ are mitochondrial rich $2 \mathrm{~A}$ muscle fibers. Since by acidic ATPase reaction we show below that there are very few to none slow type 1 muscle fibers in this area, the remaining $69 \%$ of the fibers present in this region are of Type $2 \mathrm{~B}$. In the deep region the SM muscle (right part of Fig. 1B), the type 1 (slow contracting) muscle fibers identified by positive acidic 


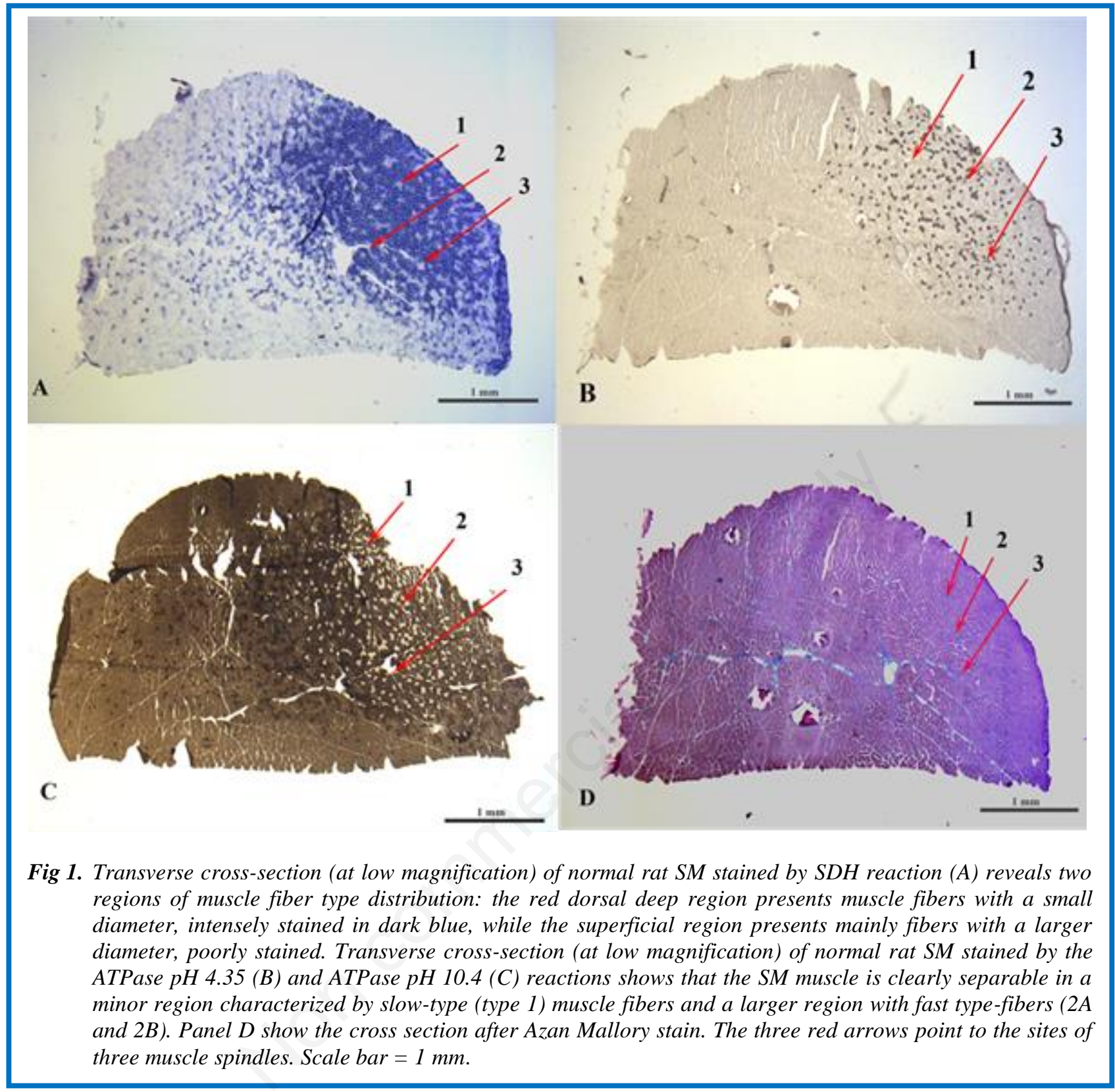

ATPase ( $\mathrm{pH} 4.35$, Fig.1B), represents only the $25 \%$ of the fibers. Indeed, though intermixed with Type $2 \mathrm{~A}$ (Acidic ATPase- negative, but SDH-rich muscle fibers) the slow type muscle fibers (i.e., those positive with the acidic ATPase, $\mathrm{pH}$ 4.35) are restricted in the deep region of the SM muscle. Therefore, $75 \%$ of the muscle fibers in the deep region are of the fast contracting types $(48 \%$ $2 \mathrm{~A}$ and $27 \% 2 \mathrm{~B}$ ). In the superficial region (left part of the cryosection) the acidic ATPase ( $\mathrm{pH}$ 4.35) technique reveals that type 1 muscle fibers are absent and that the type $2 \mathrm{~A}$ muscle fibers are decrease to $20 \%$. The vast majority of the muscle fibers present in the superficial region of are of the 2B type $69 \% \mathrm{SDH}$ - negative fibers or $80 \%$ when counted in the acidic ATPase $\mathrm{pH} 4.35$ ). Panel $\mathrm{C}$ of Fig. 1 show the pattern of staining after Alkaline ATPase reaction. The vast majority of the muscle fibers show an intermediate or strong reaction. Only in the right portion of the section sparse negative muscle fibers are present, confirming the conclusions based on the sections stained by Acidic ATPase reaction (Fig. 1, B). Rat SM muscle is composed of 2B muscle fibers that are easily recognizable because of their larger size, and 1 and 2A muscle fibers which are smaller. As expected, the acidic ATPase ( $\mathrm{pH} 4.35)$ negative and SDH-negative $2 \mathrm{~B}$ type myofibers are the largest among the three types of muscle fibers detected in rat SM muscle (Table 1). In the superficial region, based on acid ATPase ( $\mathrm{pH} 4.35$ ), the $2 \mathrm{~B}$ fiber sizes average to $47.6 \mu \mathrm{m}$ and the $2 \mathrm{~A}$ fiber size to $35.3 \mu \mathrm{m}$. In the dorsal deep red region, the type 1 fibers (slow contracting) are the smallest at an average of $29.2 \mu \mathrm{m}$; the $2 \mathrm{~A}$ and $2 \mathrm{~B}$ fiber size are $36.8 \mu \mathrm{m}$ 


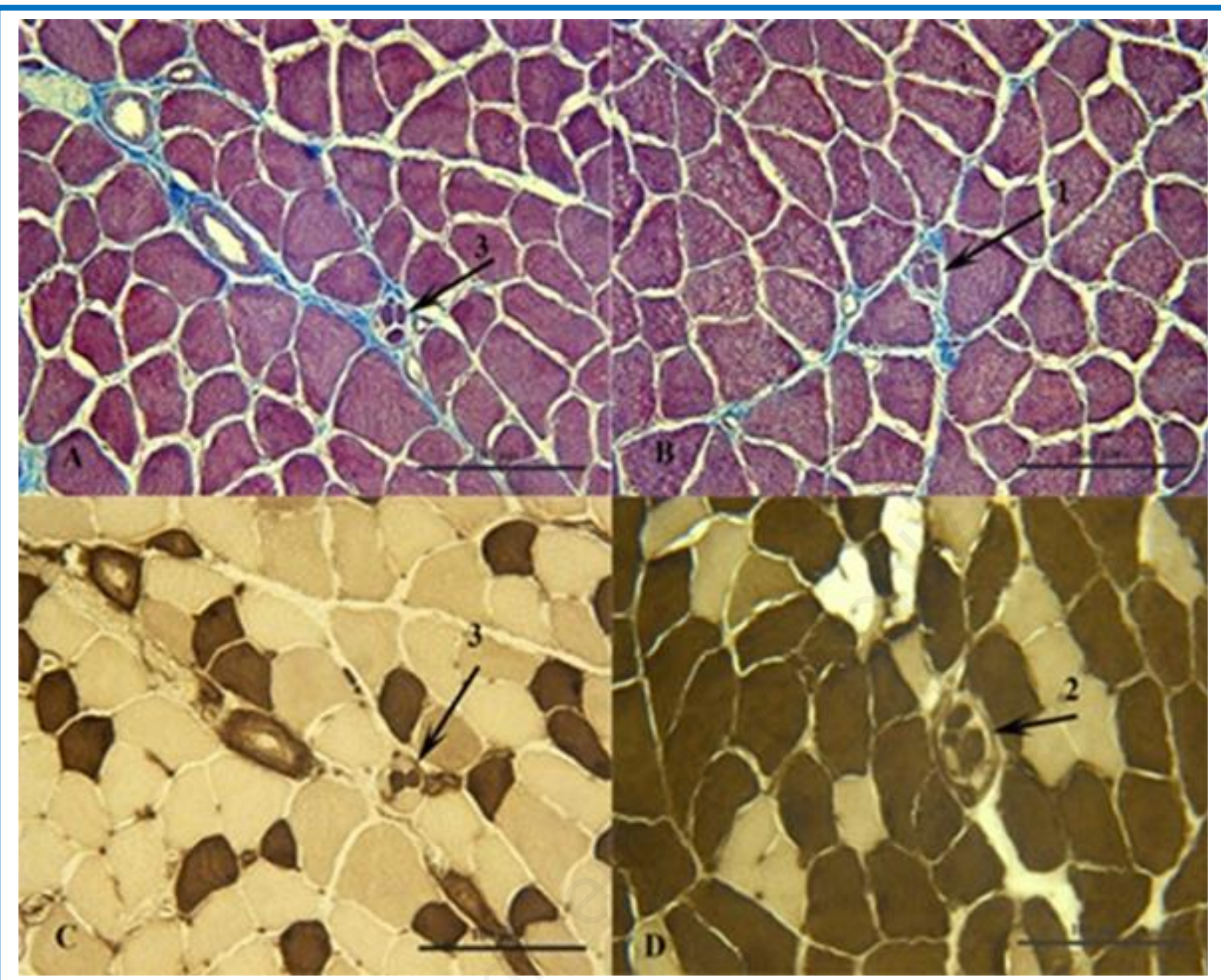

Fig 2. Transverse cross-sections (at high magnification) of normal rat SM stained by Azan Mallory (A and B), ATPase, $p H 4.35$ reaction $(C)$ or ATPase $p H 10.4$ (D) reactions. Panels A and B of Figure 2 are taken from the serial section stained by Azan Mallory and correspond to the arrow 1 and 3 indicated in the panels of figure 1. Panel C of Figure 2 is a magnification of a serial section after reaction with the Acidic ATPase ( $p H$ 4.35), while the panel D of Fig. 2 is from a serial section stained by Alkaline ATPase reaction (pH 10.4). In the sections harvested from the medial third of the rat SM muscle (Figure 1), we counted around 10,000 muscle fibers (Table 1). Note that the intrafusal muscle fibers (arrows) have smaller diameters than the normal muscle fibers. Contractile proteins of the intrafusal muscle fibers react either positive or negative with both ATPase reactions. Scale bar $100 \mu \mathrm{m}$.

on average. Based upon mitochondrial enzyme activities (i.e., the SDH reaction), the $2 \mathrm{~B}$ fibers (recognizable also as the largest ones) are $53.3 \mu \mathrm{m}$ on average in the superficial region; these fibers are $41.1 \mu \mathrm{m}$ on average in the deep region. The cumulative type 1 and $2 \mathrm{~A}$ muscle fibers average to $35.8 \mu \mathrm{m}$ in the superficial ventral region and to an average of $33.8 \mu \mathrm{m}$ in the deep region. The peculiarity of the rat SM muscle is that it presents with two distinct regions: 1) a ventral white superficial region that is characterized by a very heterogeneous distribution of muscle fiber types, having a large predominance of the type 2B fibers and a progressive enrichment of 2A fibers toward the dorsal deep red region; and 2) the dorsal deep red region that does not contain $2 \mathrm{~B}$ fibers, but an increasing number of type $2 \mathrm{~A}$ fibers, and type 1 muscle fibers that are detected only in the dorsal deep red region (Fig 1, A, B, C and Tables 1 and 2). Three muscle spindle are visible in the sections utilized for this report. Note that they are present only in the right region of the sections, that is the part easily recognized in fresh muscles since the oxidative muscle fibers (muscle fiber type 1 and 2A) have a very high content of myoglobin. ${ }^{11}$ Panel D of Figure 1 shows a serial section stained by Azan Mallory. The red stain is due to the contractile proteins of the muscle fibers, while the blue stain is due to the endomysium, i.e., to the loose collagen proteins. Panels $\mathrm{A}$ and $\mathrm{B}$ of Figure 2 are taken from the serial section stained by Azan Mallory and correspond to the arrow 1 
Table 1. Muscle fiber diameter ( $\mu m+/-S D)$ in dorsal deep or ventral superficial portions of rat SM muscle stained by SDH or ATPase pH 4.35 reactions.

\begin{tabular}{lll}
\hline & Dorsal deep portion & Ventral superficial portion \\
\hline SDH reaction & & \\
\hline Fiber Type 1+2A & $33.8 \pm 7.5$ & $35.8 \pm 9.2$ \\
Fiber Type 2B & $41.1 \pm 9.2$ & $53.3 \pm 10.8$ \\
\hline
\end{tabular}

ATPase $\mathrm{pH} 4.35$ reaction

\begin{tabular}{|c|c|c|c|}
\hline Fiber Type 1 & $29.2 \pm 6.5$ & Fiber Type 2A & $35.25 \pm 8.5$ \\
\hline Fiber Type $2 \mathrm{~A}+2 \mathrm{~B}$ & $36.8 \pm 9.2$ & Fiber Type 2B & $47.6 \pm 11.9$ \\
\hline $\begin{array}{l}\text { Muscle fibers (n.) } \\
\text { Total muscle fibers (n.) }\end{array}$ & 5802 & 4179 & \\
\hline
\end{tabular}

and 3 indicated in the panels of figure 1. Panel $\mathrm{C}$ of Figure 2 is a magnification of the serial section after reaction with the Acidic ATPase $(\mathrm{pH} 4.35)$, while the panel D of Fig. 2 is from a serial section stained by Alkaline ATPase reaction ( $\mathrm{pH}$ 10.4). The intrafusal muscle fibers have much smaller diameters than the normal muscle fibers. Indeed, the whole muscle spindle has the size of a single normal muscle fiber. Contractile proteins of the intrafusal muscle fibers react either positive or negative with both ATPase reactions. The fibrous capsule of the muscle spindle is recognized after Alkaline ATPase reaction (Panel D of Fig. 2), while being stained in blue by Azan Mallory (Panel A and B of Fig. 2). Previous studies have also stressed the usefulness of similar trichromic staining in studying human aging and pathology of the muscle spindles. ${ }^{12,13}$ To study fibrosis of the muscle spindles, the gold standard is imaging by electron microscopy,${ }^{14}$ but the paucity of the spindles in the skeletal muscles make very laborious this kind of morphometric analysis. One of the advantages of the SM muscle in experimental translational myology studies in rodents is that the muscle spindles are present only in the dorsal red portion of the muscle, thus, restricting at the macroscopic level the amount of muscle to be analysed. The topographic relation of muscle spindles to portion of fascicles composed mainly of type $2 \mathrm{~A}$ and type 1 extrafusal muscle fibers (the dorsal red portion) has been shown in several limb, ${ }^{15}$ and masticatory muscles, ${ }^{16}$ specifically in the sternomastoid, clavomastoid and clavotrapetious of the rat. ${ }^{17-19}$ Yellin

Table 2. Number and percentage muscle fibers in the dorsal deep or ventral superficial portions, stained by SDH or ATPase $\mathrm{pH} 4.35$ reactions

Dorsal deep portion $\quad$ Ventral superficial portion

SDH reaction

\begin{tabular}{lcc}
\hline Fiber Types 1+2A & $72.9 \%$ & $31.1 \%$ \\
Fiber Type 2B & $27.1 \%$ & $68.9 \%$ \\
\hline
\end{tabular}

ATPase $\mathrm{pH} 4.35$ reaction

Fiber Type 1

Fiber Type 2A + 2B
$24.5 \%$

$75.5 \%$
Fiber Type 2A

Fiber Type 2B
$20.3 \%$

$79.7 \%$ 

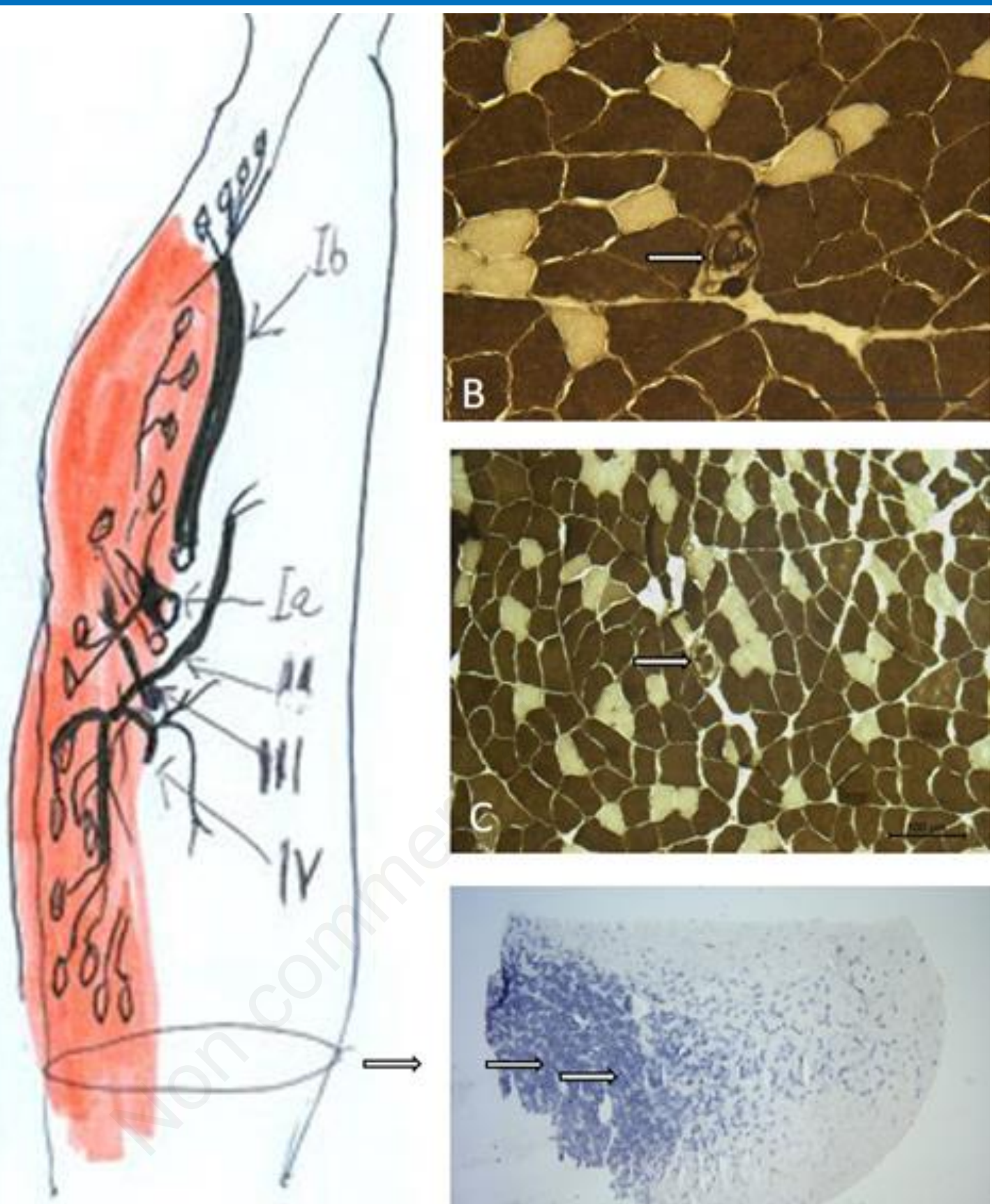

A

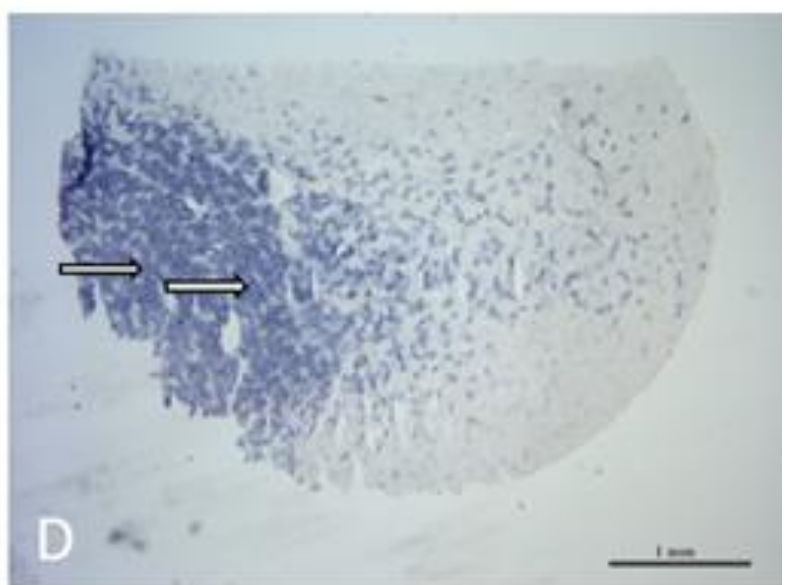

Fig. 3 A, Distribution of muscle spindles in the rat's sternomastoid muscle. The red portion of the SM, where the majority of sensory nerve fibers and of the muscle spindles (fusiform structures) are confined, is located in the ventral portion of the SM muscle. ${ }^{1}$ Around 20 muscle spindles are distributed exclusively along the antero-posterior surface of the red dorsal portion of the SM (arrows in B to D). Muscle spindles are easily recognisable from the other muscle fibers, based on their smaller size and the fibrous capsule of the spindle. The sternomastoid nerve is also indicated: Ia, Afferent nerve endings innervating muscle spindles; Ib, Afferent nerve endings of the tendon Golgi organs; II, Nerve motor fibres innervating muscle spindle; III and IV, unmyelinated sensory fibres innervate mainly the muscle fibers of the red portion of the SM muscle. The line represents the section plane of the images in B to D. B, ATPase pH 10.4. C, ATPase pH 10.4, white arrows indicate muscle spindles in the cross sections, showing variable ATPase staining properties of the intrafusal muscle fibers. D, SDH reaction, Oxidative muscle fibers 
representative of those motor units most readily recruited and thus most frequently used during less than maximal efforts. ${ }^{15,20}$ Evidence of substantial difference in daily activation of slow and fast motor units was also sumarized by Terje Lømo in a recent review ${ }^{21}$ discussing his pioneering Nature 1985 paper. $^{22}$ Thus, muscle spindles are preferentially located within the muscle in order to meet precise regulatory functions in those motor units that are frequently active and thus need frequent and fine regulation by the muscle spindles trough the Sternomastoid nerve (Figure 3 ). The absence of muscle spindle in the white portion of the muscle, where almost only type $2 \mathrm{~B}$ fast muscle fibers are restricted, suggests that the tendon Golgi organs regulate contraction of the powerfull fast type $2 \mathrm{~B}$ motor units. Innervation by the sternomastoid nerve and distribution of muscle spindles in the rat SM muscle suggest that the SM dorsal deep red portion as its sensory compartment, as far as its contents of slow fatigue resistant muscle fibers are concerned. ${ }^{1} \mathrm{On}$ the other hand, the muscle spindles are connected to the whole muscle perimysium, whose properties influence muscle stiffness and muscle spindle response to muscle stretching. If the perimysium is rigid, the muscle spindles and the tendon Golgi organs are unable to change their length, and consequently they cannot be activated..$^{23}$ Therefore, the regulation of muscle tone will be compromised and the contraction of the extrafusal muscle fibers will be altered even if the alpha motor neurons stimulate properly the muscle fibers. These changes result in decrease of muscle force, limitation of movement and altered postural control. Mense affirms that fascia disorders distort information sent by the spindles to the central nervous system, interfering with coordinated movements; specifically, spindle afferents Ia fibers are very sensitive to perimysium modifications, thus changing their discharge frequency. ${ }^{24,25}$ When perimysium is modified, as Järvinen et al. have demonstrated after limb immobilization, ${ }^{26}$ muscle spindles connected to perimysium will not function correctly. If the perimysium is altered due trauma, poor posture, post-surgery, or overuse, the inhibition of normal spindle cell stretching could result in abnormal feedback to the central nervous system. Muscle spindles, indeed, inform the central nervous system of the continually changing status of muscle tone, movement, loss of normal elasticity, position of body parts, and length of muscles.

We plan to use new imaging processes to validate present data with future analyses of muscle plasticity of the SM muscle in different experimental settings, specifically in the field of histological analyses, ${ }^{27-36}$ and clinical imaging. ${ }^{37-43}$ Machine-learning algorithms (Deep Neural Networks) have proven to be very powerful methods for automatic image segmentation, ${ }^{44}$ and this approach will be also chosen for our future analyses. In conclusion, based on present and previous data, ${ }^{1,2}$ experimental models will provide conclusive results regarding percent changes among the different fiber types and position of muscle spindles if morphometry of whole cross-sections is performed, in particular for studies of muscle plasticity in diseased muscles. ${ }^{45-62}$ In case of SM muscle biopsy from large mammals, only evaluation of sizes of the different muscle fiber types may be performed without incurring in systematic errors related to sampling. Nonetheless, histology, histochemistry and electron microscopy morphometry will provide sound quantitative evidence in translational studies of muscle plasticity.

\section{List of acronyms}

SM - sternomastoid muscle

SDH - Succinate dehydrogenase reaction

\section{Author's contributions}

Authors equally contributed to the manuscript.

\section{Acknowledgments}

BR and SZ thanks for support A\&C M-C Foundation for Translational Myology, Padova, Italy.

\section{Funding}

DC is funded by AFM (2017-20603), ANR (2013J13R191), EFEM 2016, IBPS (2014), NIH (20131R01CA108857-01 subcontractor) and UPMC Emergence (2011-EME1115).

\section{Conflict of Interest}

The authors report no conflicts of interests.

\section{Ethical Publication Statement}

We confirm that we have read the Journal's position on issues involved in ethical publication and affirm that this report is consistent with those guidelines.

\section{Corresponding Author}

Walter Giuriati, Interdepartmental Reseach Centre of Myology, c/o Department of Biomedical Sciences, University of Padova, Italy.

E-mail: walter.giuriati@unipd.it

\section{E-mails of co-authors}

Barbara Ravara: barbara.ravara@unipd.it

Andrea.Porzionato: andrea.porzionato@unipd.it

Giovanna Albertin: giovanna.albertin@unipd.it

Carla Stecco: carla.stecco@unipd.it

Veronica Macchi: veronica.macchi@unipd.it

Raffaele De Caro: raffaele.decaro@unipd.it

Chiara Gomiero: chiara.gomiero@unipd.it

Tiziana Martinello: tiziana.martinello@gmail.com

Marco Patruno: marco.pat@unipd.it

Dario Coletti: dario.coletti@uniroma1.it

Sandra Zampieri: sanzamp@unipd.it

Alessandra Nori: alessandra.nori@unipd.it

\section{References}

1. Zenker W, Sandoz PA, Neuhuber W. The distribution of anterogradely labelled I-IV primary afferents in histochemically defined compartments 
of the rat's sternomastoid muscle. Anat Embryol 1998;177:235-43.

2. Polican Ciena A, Yokomito de Almeida SR, de Matos Alves PH, et al. Histological and ultrastructural changes of sternomastoid muscle in aged Wistar rats. Micron 2011;42:871-6.

3. Hiroux C, Vandoorne T, Koppo K,et al. Physical Activity Counteracts Tumor Cell Growth in Colon Carcinoma C26-Injected Muscles: An Interim Report. Eur J Transl Myol 2016;26:5958. doi: 10.4081/ejtm.2016.5958. eCollection 2016 Jun 13.

4. Tasic D, Dimov D, Gligorijevic J, et al. Muscle fibre types and fibre morphometry in the tibialis posterior and anterior of the rat: a comparative study. Medicine and Biology 2003;10:16-21.

5. Coletti D, Daou N, Hassani M, et al. Serum Response Factor in Muscle Tissues: From Development to Ageing. Eur J Transl Myol 2016;26:6008. doi: 10.4081/ejtm.2016.6008. eCollection 2016 Jun 13.

6. Kern H, Loefler S, Hofer C, et al. FES Training in Aging: interim results show statistically significant improvements in mobility and muscle fiber size. Eur J Transl Myol 2012;22:61-7.

7. Brooke MH, Kaiser KK. J. Some comments on the histochemical caracterization of muscle adenosin triphosphatase. Histochem Cytochem 1969;17:4312.

8. Brooke MH, Kaiser KK. Muscle fiber types: How many and what kind? Arch Neurol 1970 23; 369. 79.

9. Guth L, Samaha FJ. Qualitative differences between actomyosin ATPase of slow and fast mammalian muscles Exp Neurol 1969;25:139-52.

10. Guth L, Samaha FJ. Procedure for the histochemical demonstration of actomyosin ATPase. Exp Neurol 1970;28: 365-7.

11. Hammalinen N, Pette D. The histochemical profiles of fast fibers IIB, IIA in skeletal muscle of mouse, rat and rabbit. Histochem Cytochem 1993;41:73343.

12. Boyd-Clark LC, Briggs CA, Galea MP. Muscle spindle distribution, morphology, and density in longus colli and multifidus muscles of the cervical spine. Spine 2002;27:694-701.

13. De Reuck J. The pathology of the human muscle spindle. A light microscopic, biometric and histochemical study. Acta Neuropath 1974;30:4350.

14. Ovalle WK, Dow PR. Morphological aspects of the muscle spindle capsule and its functional significance.In: The Muscle Spindle, Boyd IA, Gladden MH, eds. Stockton Press, New York. 1985: pp. 23-28.

15. Yellin H. A histochemical study of muscle spindles and their relationship to extrafusal fiber types in the rat. Am J anat 1969;125:31-45.
16. Maier A. Occurrence and distribution of muscle spindles in masticatory and suprahyoid muscles of the rat. Am J Anat 1979;155:483-506.

17. Grimm M. On the problem of irregular distribution of muscle spindles. Folia Morphol 1972;20:119-20.

18. Gottschall J, Zenker W, Neuhuber W, Mysicka A, Müntener M. The sternomastoid muscle of the rat and its innervation. Muscle fiber composition, perikarya and axons of efferent and afferent neurons. Anat Embryol (Berl). 1980;160(3):285300.

19. Brichta AM, Callister RJ, Peterson EH. Quantitative analysis of cervical musculature in rats: histochemical composition and motor pool organization. I. Muscles of the spinal accessory complex. J Comp Neurol. 1987 Jan 15;255(3):35168.

20. Henneman E, Olson CB. Relations between structure and function in the design of skeletal muscles. Neurophysiol. 1965 May;28:581-98.

21. Lømo T. The Response of Denervated Muscle to Long-Term Stimulation (1985, Revisited here in 2014). Eur J Transl Myol 2014;24:3294. doi: 10.4081/ejtm.2014.3294. eCollection 2014 Mar 31.

22. Hennig R, Lømo T. Firing patterns of motor units in normal rats. Nature 1985;314:164-6.

23. Wilke J, Schleip R, Klingler W, Stecco C. The Lumbodorsal Fascia as a Potential Source of Low Back Pain: A Narrative Review. Biomed Res Int 2017;2017:5349620. doi: 10.1155/2017/5349620.

24. Mense S. Peripheral and central mechanisms of myofascial pain. Presented at Pittsburg Conference on Myofascial Component of Musculoskeletal Pain. University of Pittsburg, May 7-8, 2011.

25. Tesarz J, Hoheisel U, Wiedenhöfer B, Mense S. Sensory innervation of the thoracolumbar fascia in rats and humans. Neuroscience 2011;194:302-8. doi: 10.1016/j.neuroscience.2011.07.066.

26. Järvinen TA, Józsa L, Kannus P, et al. Organization and distribution of intramuscular connective tissue in normal and immobilized skeletal muscles. An immunohistochemical, polarization and scanning electron microscopic study. J Muscle Res Cell Motil 2002;23:245-54.

27. Seene T, Umnova M, Kaasik P. Morphological peculiarities of neuromuscular junctions among different fiber types: Effect of exercise. Eur J Transl Myol. 2017 Jun 27;27(3):6708. doi: 10.4081/ejtm.2017.6708. eCollection 2017 Jun 27.

28. Samiee F, Zarrindast MR. Effect of electrical stimulation on motor nerve regeneration in sciatic nerve ligated-mice. Eur J Transl Myol. 2017 Sep 20;27(3):6488. doi: 10.4081/ejtm.2017.6488. eCollection 2017 Jun 27.

29. Power GA, Dalton BH, Gilmore KJ, et al. Maintaining Motor Units into Old Age: Running the Final Common Pathway. Eur J Transl Myol 


\section{Rat sternomastoid muscle spindles}

Eur J Transl Myol 28 (4): 376-385, 2018

2017;27(1):6597. doi: 10.4081/ejtm.2017.6597. eCollection 2017 Feb 24.

30. Pigna E, Greco E, Morozzi G, et al. Denervation does not Induce Muscle Atrophy Through Oxidative Stress. Eur J Transl Myol 2017;27(1):6406. doi: 10.4081/ejtm.2017.6406. eCollection 2017 Feb 24.

31. Pette D, Vrbová G. The Contribution of Neuromuscular Stimulation in Elucidating Muscle Plasticity Revisited. Eur J Transl Myol. 2017 Feb 24;27(1):6368. doi: 10.4081/ejtm.2017.6368. eCollection 2017 Feb 24. Review.

32. Carotenuto F, Coletti D, Di Nardo P, Teodori L. $\alpha$ Linolenic Acid Reduces TNF-Induced Apoptosis in C2C12 Myoblasts by Regulating Expression of Apoptotic Proteins. Eur J Transl Myol 2016;26(4):6033. doi: 10.4081/ejtm.2016.6033. eCollection 2016 Sep 15.22.

33. Riebold B, Nahrstaedt H, Schultheiss C, et al. Multisensor Classification System for Triggering FES in Order to Support Voluntary Swallowing. Eur J Transl Myol 2016;26(4):6224. doi: 10.4081/ejtm.2016.6224. eCollection 2016 Sep 15.33 .

34. Stratton K, Faghri PD. Electrically and HybridInduced Muscle Activations: Effects of Muscle Size and Fiber Type. Eur J Transl Myol 2016;26(3):6163. eCollection 2016 Jun 13.

35. Willand MP. Electrical Stimulation Enhances Reinnervation After Nerve Injury. Eur J Transl Myol 2015;25(4):243-8. doi: 10.4081/ejtm.2015. 5243. eCollection 2015 Aug 24. Review.

36. Zampieri S, Mosole S, Löfler S, et al. Physical Exercise in Aging: Nine Weeks of Leg Press or Electrical Stimulation Training in 70 Years Old Sedentary Elderly People. Eur J Transl Myol 2015;25(4):237-42. doi: 10.4081/ejtm.2015.5374. eCollection 2015 Aug 24. Review.

37. Edmunds KJ, Árnadóttir Í, Gíslason MK, Carraro U, Gargiulo P. Nonlinear Trimodal Regression Analysis of Radiodensitometric Distributions to Quantify Sarcopenic and Sequelae Muscle Degeneration. Comput Math Methods Med. 2016;2016:8932950. doi: 10.1155/2016/8932950. Epub 2016 Dec 27.

38. Albertin G, Hofer C, Zampieri S, Vogelauer M, Löfler S, Ravara B, Guidolin D, Fede C, Incendi D, Porzionato A, De Caro R, Baba A, Marcante A, Piccione F, Gargiulo P, Pond A, Carraro U, Kern H. In complete SCI patients, long-term functional electrical stimulation of permanent denervated muscles increases epidermis thickness. Neurol Res. 2018 Apr;40(4):277-282. doi: 10.1080/01616412. 2018.1436877. Epub 2018 Feb 15.

39. Kern H, Hofer C, Loefler S, Zampieri S, Gargiulo P, Baba A, Marcante A, Piccione F, Pond A, Carraro U. Atrophy, ultra-structural disorders, severe atrophy and degeneration of denervated human muscle in SCI and Aging. Implications for their recovery by Functional Electrical Stimulation, updated 2017. Neurol Res. 2017 Jul;39(7):660-666. doi: 10.1080/01616412.2017.1314906. Epub 2017 Apr 13. Review.

40. Edmunds K, Gislason M, Sigurdsson S, Gudnason V, Harris T, Carraro U, Gargiulo P. Advanced quantitative methods in correlating sarcopenic muscle degeneration wqith lower extremity function biometrics and comorbidities. PLos ONE 13(3):

e0193241. https://doi.org/10.1371/journal.pone.0193241

41. Carraro U, Edmunds KJ, Gargiulo P. 3D False Color Computed Tomography for Diagnosis and Follow-Up of Permanent Denervated Human Muscles Submitted to Home-Based Functional Electrical Stimulation. Eur J Transl Myol 2015;25(2):5133. doi: 10.4081/ejtm.2015.5133. eCollection 2015 Mar 11. Review.

42. Ortolan P, Zanato R, Coran A, et al. Role of Radiologic Imaging in Genetic and Acquired Neuromuscular Disorders. Eur J Transl Myol 2015;25(2):5014. doi: 10.4081/ejtm.2015.5014. eCollection 2015 Mar 11. Review.

43. Magnússon B, Pétursson $\mathrm{P}$, Edmunds $\mathrm{K}$, et al. Improving Planning and Post-Operative Assessment for Total Hip Arthroplasty. Eur J Transl Myol 2015;25(2):4913. doi: 10.4081/ejtm.2015.4913. eCollection 2015 Mar 11.

44. Ciresan DC, Giusti A, Gambardella LM, Schmidhuber J. Mitosis detection in breast cancer histology images with deep neural networks. Med Image Comput Assist Interv 2013;16:411-8.

45. Barberi L, Scicchitano BM, Musaro A. Molecular and Cellular Mechanisms of Muscle Aging and Sarcopenia and Effects of Electrical Stimulation in Seniors. Eur J Transl Myol 2015;25(4):231-6. doi: 10.4081/ejtm.2015.5227. eCollection 2015 Aug 24. Review.

46. Ravara B, Gobbo V, Carraro U, et al. Functional Electrical Stimulation as a Safe and Effective Treatment for Equine Epaxial Muscle Spasms: Clinical Evaluations and Histochemical Morphometry of Mitochondria in Muscle Biopsies. Eur J Transl Myol 2015;25(2):4910. doi: 10.4081/ejtm.2015.4910. eCollection 2015 Mar 11.

47. Costa A, Rossi E, Scicchitano BM, et al. Neurohypophyseal Hormones: Novel Actors of Striated Muscle Development and Homeostasis. Eur J Transl Myol 2014;24(3):3790. doi: 10.4081/ejtm.2014.3790. eCollection 2014 Sep 23. Review.

48. Hockerman GH, Dethrow NM, Hameed S, et al. The Ubr2 Gene is Expressed in Skeletal Muscle Atrophying as a Result of Hind Limb Suspension, but not Merg1a Expression Alone. Eur J Transl Myol. 2014;24(3):3319. doi: 10.4081/ejtm.2014.3319. eCollection 2014 Sep 23. 
49. Carlson BM. The Biology of Long-Term Denervated Skeletal Muscle. Eur J Transl Myol. 2014 Mar 27;24:3293. doi: 10.4081/ejtm.2014.3293. eCollection 2014 Mar 31.

50. Paillard T. Muscle plasticity of aged subjects in response to electrical stimulation training and inversion and/or limitation of the sarcopenic process. Ageing Research Review 2018;46:1-13. doi.org/10.1016/j.arr.2018.05.002.

51. Carlson BM. The Biology of Long-Term Denervated Skeletal Muscle. Eur J Transl Myol. 2014 Mar 27;24:3293. doi: 10.4081/ejtm.2014.3293. eCollection 2014 Mar 31.

52. Kern H, Carraro U. Home-Based Functional Electrical Stimulation for LongTerm Denervated Human Muscle: History, Basics, Results and Perspectives of the Vienna Rehabilitation Strategy. Eur J Transl Myol 2014;24(1):3296. doi: 10.4081/ejtm.2014.3296. eCollection 2014 Mar 31.

53. Carraro U, Boncompagni S, Gobbo V, et al. Persistent Muscle Fiber Regeneration in Long Term Denervation. Past, Present, Future. Eur J Transl Myol 2015;25(2):4832. doi: 10.4081/ejtm.2015. 4832. eCollection 2015 Mar 11. Review.

54. Barberi L, Scicchitano BM, Musaro A Molecular and cellular mechanisms of muscle aging and sarcopenia and effects of electrical stimulation in seniors. Eur J Transl Myol 2015;25(4):231-6. doi: 10.4081/ejtm.2015.5227. eCollection 2015 Aug 24. Review.

55. Mosole S, Carraro U, Kern H, Loefler S, Zampieri S. Use it or Lose It: Tonic Activity of Slow Motoneurons Promotes Their Survival and Preferentially Increases Slow Fiber-Type Groupings in Muscles of Old Lifelong Recreational Sportsmen. Eur J Transl Myol 2016;26(4):5972. doi: 10.4081/ejtm.2016.5972. eCollection 2016 Sep 15.

56. Edmunds KJ, Gíslason MK, Arnadottir ID, et al. Quantitative Computed Tomography and Image Analysis for Advanced Muscle Assessment. Eur J Transl Myol 2016 Jun 22;26(2):6015. doi: 10.4081/ejtm.2016.6015. eCollection 2016 Jun 13.

57. Sajer S. Mobility disorders and pain, interrelations that need new research concepts and advanced clinical commitments. Eur J Transl Myol 2017;27(4):7179. doi: 10.4081/ejtm.2017.7179. eCollection 2017 Dec 5.

58. Ricci G, Simoncini C, Baldanzi S, Siciliano G. Opportunities and potential applications from healthcare technologies to assist motor activity in metabolic myopathies. Biol Eng Med 2018;3(3):14. doi: 10.15761/BEM.1000S1001.

59. Hofer C, Loefler S, Kern H, et al. Two years of FES training improves muscle fibers of thigh muscles in long-term thoracic level-complete spinal cord injury. Biol Eng Med 2018;3(3):1-5. doi: 10.15761/BEM.1000S1002.

60. Carraro U, Albertin G, Gargiulo P, et al. Muscle and skin improve by home-based FES and fullbody inbed gym. Biol Eng Med 2018;3(3):1-4. doi: 10.15761/BEM.1000S1003.

61. Coletti D. Exercise against tumor- and chemotherapy-induced muscle wasting. Biol Eng Med, 2018; 3(3):1-5 doi: 10.15761/BEM. $1000 \mathrm{~S} 1004$.

62. Masiero S, Musumeci A. Rehabilitation medicine for elderly patients, a further note. Biol Eng Med, 2018; 3(3):1-2 doi: 10.15761/BEM.1000S1006.

Received for publication: 30/10/2018

Accepted for publication: 13/11/2018 